\title{
Differential expression of the long and short form of CDK2 in glioblastoma tumors.
}

Shahan Mamoor, MS1

1shahanmamoor@gmail.com

Glioblastoma is a cancer of the brain with poor survival outcomes and limited treatment options $(1,2)$. CDK inhibitors have recently been introduced to the oncology market with multiple CDK inhibitor options in metastatic breast cancer, including CDK4/6 inhibitor palbociclib (3). We performed global gene expression profiling of glioblastoma tumors, comparing the tumors transcriptomes from patients with glioblastoma multiform with that of the non-affected tissues using published microarray datasets $(4,5)$.

Transcripts encoded by the CDK2 gene, including a long form of the CDK2 transcript, CDK2L, were both among the transcripts most differentially expressed in patients with glioblastoma. CDK2 and its associated regulatory partners may be relevant targets of study in glioblastoma multiforme, a disease with zero targeted treatments available.

Keywords: glioblastoma, differential gene expression analysis, systems oncology, targeted cancer therapeutics, tumor transcriptome analysis, CDK2, CDK2L. 


\section{Introduction}

Systems oncology presupposed nothing and instead queries all of the genes, gene transcripts, or gene products produced in a cell, to ask any number of questions: what the most significantly different RNAs produced between a tumor cell and its benign counterpart (7), or what are the genes most necessary for a cancer cells survival, and equally as important, which of these genes that are required for a cancer cells survival are not necessary, or less necessary for normal, non-transformed cells (8).

Glioblastoma is the most common brain cancer in adults $(1,2)$. The standard of care includes surgery, if possible, to debulk the tumor, chemotherapy including temozolomide and an implantable wafer containing a mustard-based chemotherapy called polifeprosan 20 with carmustine, as well as radiation therapy (9-12). These treatments provide a 15 month median survival rate at diagnosis $(1,2)$. There is a dire need for the development of novel therapeutics for the treatment of glioblastoma, and the development of these drugs requires a basic understanding of the transcriptional behavior of the cells that make up glioblastoma tumors.

In this study, we performed global differential gene expression analysis to compare the transcriptomes of 17 tumors isolated from patients diagnosed with glioblastoma multiform to that of the non-affected brain transcriptome using a published dataset (4). We cross referenced these findings with a separate analysis of, comparing benign kidney tissue as control to glioblastoma tissues (5). These analyses revealed that the cyclin dependent kinase CDK2 and the long isoform of CDK2, CDK2L, were differentially expressed by primary glioblastoma tumors from humans. 


\section{Methods}

GSE116520 (4) and GSE122498 (5) were used for this differential gene expression analysis, in conjunction with GEO2R. The transcriptional data from GSE116520 contains tumor RNA from 17 patients diagnosed with glioblastoma

multiform and brain tissue RNA from 8 controls, was generated by Kruthika et al. using Illumina HumanHT-12 V4.0 expression beadchip technology. GSE122498 was generated using Affymetrix Human Genome U133 Plus 2.0 Array with n=1 normal kidney tissue and $n=16$ glioblastoma tumors. The $p$-value adjustment option was set to "Benjamini and Hochberg", application of log transformation to data was set to "Auto-detect" and the NCBI generated category of platform annotation was used. Statistical tests to compare the significance of RNA expression values between tumor and control groups was performed using a two-tailed, unpaired t-test with Welch's correction (PRISM 8.1.2) (227). All genes/transcripts reported here are statistically significant with respect to global differential expression (Table 1).

\section{Results}

CDK2 and the long form of CDK2, CDK2L, are both differentially expressed in glioblastoma tumors when compared to the brain.

We performed differential gene expression analysis on a global level by comparing the transcriptomes of tumors from 17 patients diagnosed with glioblastoma to the brain transcriptome, using non-affected control brain tissue samples. We found that the cyclin dependent kinase CDK2 a longer splice variant of CDK2, CDK2L, were both among the genes most differentially expressed in glioblastoma tumors (Table 1). CDK2 was ranked 44th in terms of differential expression and CDK2L was ranked 46th, 
out of 47229 total transcripts detected and measured in the microarray data analyzed here. In both cases, this differential expression was statistically significant (Table 1; CDK2: $p=3.61 \mathrm{E}-15$; CDK2L: $p=4 \mathrm{E}-15)$.

CDK2 and CDK2L display opposite differential expression patterns in glioblastoma tumors

We obtained the RNA expression levels for each gene from each of 17 patient tumor sample, as well as from all 8 control, non-affected brain samples. We used a statistical test to compare the expression levels and determine whether the difference in expression of CDK2 and CDK2L between tumor and control groups was statistically significant. CDK2 was expressed at higher levels in glioblastoma tumors than in control brain tissue, and this difference was statistically significant (Figure $1 ; p<0.0001$ ). Interestingly, CDK2L was expressed at lower levels in glioblastoma tumors that in control brain tissue, and this difference was also statistically significant (Figure 2; $\mathrm{p}<0.0001$ ).

Differential expression of CDK2 gene in a separate glioblastoma tumor dataset.

We queried microarray data from a second study (5) to determine whether differential expression of a CDK2 transcript could be observed in an independent dataset. We found significant differential expression of CDK2 in tumors from this group of patients with glioblastoma. When sorting all transcripts measured by microarray based on change in expression between control tissue and glioblastoma tumor tissue, CDK2 ranked 7351 out of 54675 transcripts. Differential expression of CDK2 was statistically significant (Table $3 ; p=.2 .85 E-02$ ). Thus, the change of expression in CDK2 
in glioblastoma tumors in this second dataset was statistically more significant than $86.6 \%$ of the measured transcriptome.

\section{Discussion}

Systems-level analysis of glioblastoma can describe the most significant differences between the genome (DNA) and transcriptome (RNA) of the cancer compared to the tissue from which it arises, the brain (13-16). Here, we performed global differential gene expression analysis by comparing the transcriptome of glioblastoma tumors from 17 patients to the brain transcriptomes from 8 non-affected individuals as control using published microarray data. We found that the cyclin dependent kinase CDK2 and the long form of CDK2, CDK2L, were among the genes that were expressed most significantly differently between glioblastoma tumors and the brain in this dataset. We asked whether differential expression of any CDK2 transcripts could be observed in a separate microarray dataset (5), and found significantly differential expression of the short form of CDK2 when comparing glioblastoma tumors to normal kidney tissue. While perturbed expression of CDK2 has previously been reported in glioblastoma (6), we could not identify literature describing differential expression of CDK2L in the primary tumors of patients with glioblastoma.

CDK2L (otherwise referred to as CDKL2) is transcribed from the same gene as the "short" form (arm) of CDK2, and can form a complex with Cyclin A. CDK2L possesses approximately half of the kinase activity of that of CDK2 in complex with Cyclin A (17). Since CDK2L was differentially expressed in glioblastomas but at significantly lower levels in GBM tumors as compared to normal tissue, and since CDK2L possesses half the activity of CDK2 and shares some of the same substrates 
(e.g., Histone $\mathrm{H} 1$ ), the presence of CDK2L might function as an endogenous competitive inhibitor of CDK2 by sterically occupying the same CDK2 substrates but exerting only half of the kinase activity. Since CDK2 expression has been argued to provide a proliferative advantage to glioblastomas, decreased expression of an endogenous inhibitor of CDK2 activity like CDK2L would support glioblastoma growth and survival by down-regulation of an endogenous CDK inhibitor. This could be experimentally assessed in xenograft mouse models of glioblastoma $(18,19)$, and in isolated human glioblastoma patient samples ex vivo treated with CDK2 inhibitors (20-22) or with genetic depletion of CDK2 using short hairpin silencing of CDK2 delivered by lentivirus infection.

In this study, we found that CDK2 and CDK2L are differentially expressed in an opposing fashion in glioblastoma tumors relative to control, non-affected brain, with significantly higher expression of CDK2 in glioblastoma tumors that in the brain, and significantly lower expression of a different, longer CDK2 transcript, CDK2L, in glioblastoma tumors than in the brain, using published microarray data (4). We validated differential expression of the CDK2 gene in glioblastomas in a separate dataset (5). These results merit validation of these results in larger datasets, at the protein level using CDK2 antibodies, and provide rationale for assessment of whether whether modulation of CDK2L affects substrate phosphorylation of CDK2 targets, as well as glioblastoma tumor cell proliferation or survival, in vitro and in vivo, 


\section{References}

1. Davis, M.E., 2016. Glioblastoma: overview of disease and treatment. Clinical journal of oncology nursing, 20(5), p.S2.

2. Alexander, B.M. and Cloughesy, T.F., 2017. Adult glioblastoma. Journal of Clinical Oncology, 35(21), pp.2402-2409.

3. O'Leary, B., Finn, R.S. and Turner, N.C., 2016. Treating cancer with selective CDK4/6 inhibitors. Nature reviews Clinical oncology, 13(7), p.417.

4. Kruthika, B.S., Jain, R., Arivazhagan, A., Bharath, R.D., Yasha, T.C., Kondaiah, P. and Santosh, V., 2019. Transcriptome profiling reveals PDZ binding kinase as a novel biomarker in peritumoral brain zone of glioblastoma. Journal of neurooncology, 141(2), pp.315-325.

5. Hilf, N., Kuttruff-Coqui, S., Frenzel, K., Bukur, V., Stevanović, S., Gouttefangeas, C., Platten, M., Tabatabai, G., Dutoit, V., van der Burg, S.H. and Thor Straten, P., 2019. Actively personalized vaccination trial for newly diagnosed glioblastoma. Nature, 565(7738), pp.240-245.

6. Wang, J., Yang, T., Xu, G., Liu, H., Ren, C., Xie, W. and Wang, M., 2016. Cyclindependent kinase 2 promotes tumor proliferation and induces radio resistance in glioblastoma. Translational oncology, 9(6), pp.548-556.

7. Rhodes, D.R. and Chinnaiyan, A.M., 2005. Integrative analysis of the cancer transcriptome. Nature genetics, 37(6s), p.S31.

8. Schlabach, M.R., Luo, J., Solimini, N.L., Hu, G., Xu, Q., Li, M.Z., Zhao, Z., Smogorzewska, A., Sowa, M.E., Ang, X.L. and Westbrook, T.F., 2008. Cancer proliferation gene discovery through functional genomics. Science, 319(5863), pp. 620-624.

9. Stupp, R., Mason, W.P., Van Den Bent, M.J., Weller, M., Fisher, B., Taphoorn, M.J., Belanger, K., Brandes, A.A., Marosi, C., Bogdahn, U. and Curschmann, J., 2005. Radiotherapy plus concomitant and adjuvant temozolomide for glioblastoma. New England Journal of Medicine, 352(10), pp.987-996.

10. McGirt, M.J., Than, K.D., Weingart, J.D., Chaichana, K.L., Attenello, F.J., Olivi, A., Laterra, J., Kleinberg, L.R., Grossman, S.A., Brem, H. and Quiñones-Hinojosa, A., 2009. Gliadel (BCNU) wafer plus concomitant temozolomide therapy after primary resection of glioblastoma multiforme. Journal of neurosurgery, 110(3), pp.583-588. 
11. Affronti, Mary Lou, Christopher R. Heery, James E. Herndon, Jeremy N. Rich, David A. Reardon, Annick Desjardins, James J. Vredenburgh, Allan H. Friedman, Darell D. Bigner, and Henry S. Friedman. "Overall survival of newly diagnosed glioblastoma patients receiving carmustine wafers followed by radiation and concurrent temozolomide plus rotational multiagent chemotherapy." Cancer: Interdisciplinary International Journal of the American Cancer Society 115, no. 15 (2009): 3501-3511.

12. National Comprehensive Cancer Network, 2015. Clinical Practice Guidelines in Oncology: Central nervous system cancers [v. 1.2015].

13. Brennan, C.W., Verhaak, R.G., McKenna, A., Campos, B., Noushmehr, H., Salama, S.R., Zheng, S., Chakravarty, D., Sanborn, J.Z., Berman, S.H. and Beroukhim, R., 2013. The somatic genomic landscape of glioblastoma. Cell, 155(2), pp.462-477.

14. Parsons, D.W., Jones, S., Zhang, X., Lin, J.C.H., Leary, R.J., Angenendt, P., Mankoo, P., Carter, H., Siu, I.M., Gallia, G.L. and Olivi, A., 2008. An integrated genomic analysis of human glioblastoma multiforme. Science, 321(5897), pp. 1807-1812.

15. Ohgaki, H. and Kleihues, P., 2007. Genetic pathways to primary and secondary glioblastoma. The American journal of pathology, 170(5), pp.1445-1453.

16. Wang, J., Cazzato, E., Ladewig, E., Frattini, V., Rosenbloom, D.I., Zairis, S., Abate, F., Liu, Z., Elliott, O., Shin, Y.J. and Lee, J.K., 2016. Clonal evolution of glioblastoma under therapy. Nature genetics, 48(7), p.768.

17. Ellenrieder, C., Bartosch, B., Lee, G.Y.C., Murphy, M., Sweeney, C., Hergersberg, M., Carrington, M., Jaussi, R. and Hunt, T., 2001. The long form of CDK2 arises via alternative splicing and forms an active protein kinase with cyclins $\mathrm{A}$ and $\mathrm{E}$. DNA and cell biology, 20(7), pp.413-423.

18. Saito, R., Bringas, J.R., Panner, A., Tamas, M., Pieper, R.O., Berger, M.S. and Bankiewicz, K.S., 2004. Convection-enhanced delivery of tumor necrosis factorrelated apoptosis-inducing ligand with systemic administration of temozolomide prolongs survival in an intracranial glioblastoma xenograft model. Cancer research, 64(19), pp.6858-6862.

19. Giannini, C., Sarkaria, J.N., Saito, A., Uhm, J.H., Galanis, E., Carlson, B.L., Schroeder, M.A. and James, C.D., 2005. Patient tumor EGFR and PDGFRA gene amplifications retained in an invasive intracranial xenograft model of glioblastoma multiforme. Neuro-oncology, 7(2), pp.164-176.

20. Payton, M., Chung, G., Yakowec, P., Wong, A., Powers, D., Xiong, L., Zhang, N., Leal, J., Bush, T.L., Santora, V. and Askew, B., 2006. Discovery and evaluation of dual CDK1 and CDK2 inhibitors. Cancer research, 66(8), pp.4299-4308. 
21. Chohan, T.A., Qian, H., Pan, Y. and Chen, J.Z., 2015. Cyclin-dependent kinase-2 as a target for cancer therapy: progress in the development of CDK2 inhibitors as anticancer agents. Current medicinal chemistry, 22(2), pp.237-263.

22. Kawakami, M., Liu, X., Mustachio, L.M., Sekula, D.J., Hu, S., Zheng, L., RodriguezCanales, J., Mino, B., Villalobos, P.A., Behrens, C. and Wistuba, I.I., 2016.

Antineoplastic activity of the novel CDK2/9 inhibitor CCT68127 occurs via induced anaphase catastrophe and inhibition of PEA15 phosphorylation in lung cancer. 


\begin{tabular}{|c|c|c|c|c|c|c|}
\hline Rank & Gene symbol & Gene name & p-value & $\mathbf{t}$ & B & ID \\
\hline 44 & CDK2 & cyclin dependent kinase 2 & $3.61 \mathrm{E}-15$ & -17.1310438 & 24.717018 & ILMN_1665559 \\
\hline 46 & CDK2L & cyclin dependent kinase like 2 & $4 \mathrm{E}-15$ & 17.0544987 & 24.618606 & ILMN_1856533 \\
\hline
\end{tabular}

Table 1: Cyclin dependent kinase CDK2 and CDK2L are among the genes most differentially expressed in the tumors of patients with glioblastoma multiform.

Rank of differential expression, gene symbol, name of the gene whose transcript was identified as differentially expressed, $\mathrm{p}$-value with respect to global differential expression (relative to the transcriptome), t, a moderated t-statistic, and $B$, and the log-odds of differential expression between the two groups is shown (all provided by GEO2R), as well as Illumina probe ID. 


\section{CDK2}

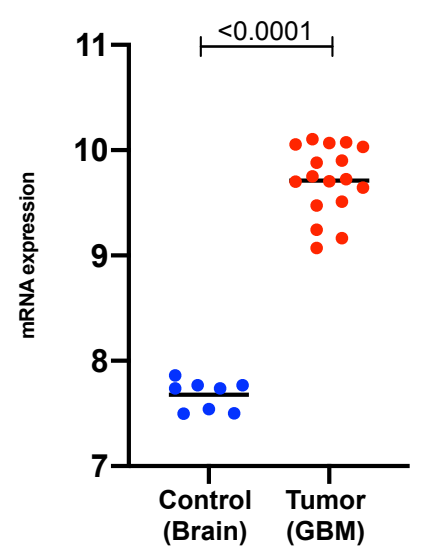

CDK2

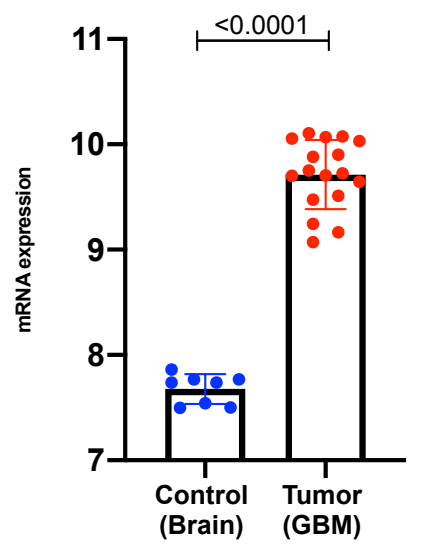

CDK2

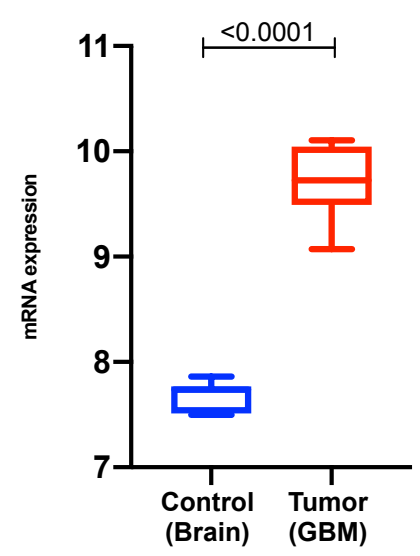

Figure 1: CDK2 is expressed at significantly higher levels in the glioblastoma tumors compared to non-affected control brain tissue.

The RNA expression value of CDK2 is depicted graphically, by dots representing RNA expression in each patient tumor or control brain tissue (left), by bar graph with standard error of the mean (SEM) (middle), or by box and whisker plot (right). The significance of difference between the mean expression values in tumor versus control group is shown on each graph as a $p$-value, determined by a two-tailed unpaired t-test with Welch's correction. 


\section{CDKL2}

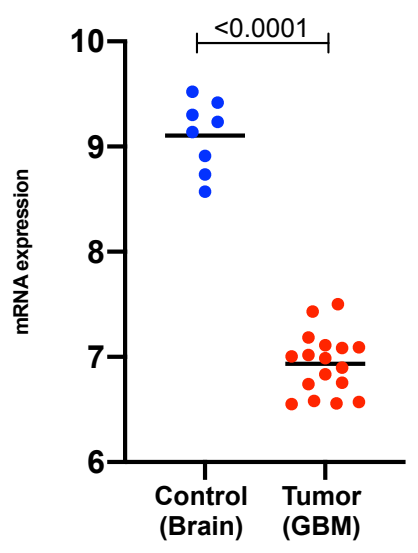

CDKL2

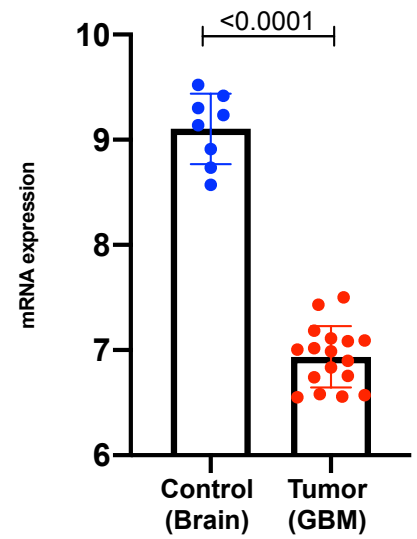

CDKL2

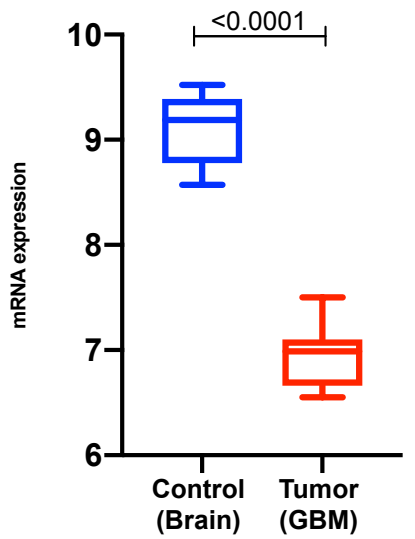

Figure 2: CDK2L is expressed at significantly lower levels in glioblastoma tumors compared to non-affected control brain tissue.

The RNA expression value of CDK2L is depicted graphically, by dots representing RNA expression in each patient tumor or control brain tissue (left), by bar graph with standard error of the mean (SEM) (middle), or by box and whisker plot (right). The significance of difference between the mean expression values in tumor versus control group is shown on each graph as a $p$-value, determined by a two-tailed unpaired t-test with Welch's correction. 


\begin{tabular}{|c|l|l|l|l|l|l|}
\hline Rank & ID & p-value & t & B & Gene & Gene name \\
\hline $\mathbf{7 3 5 1}$ & 204252_at & 2.85E-02 & 2.3765826 & -3.68091062 & CDK2 & cyclin dependent kinase 2 \\
\hline
\end{tabular}

Table 3: CDK2 is among the genes most differentially expressed in glioblastoma tumors when comparing GBM tumors to non-affected control kidney tissue.

Rank of differential expression, probe ID, p-value with respect to global differential expression (relative to the transcriptome), $t$, a moderated t-statistic, and $B$, the log-odds of differential expression between the two groups, gene and gene name is listed in this chart. 